\title{
Evaluation of Usability Dimensions of Smartphone Applications
}

\author{
Shabana Shareef ${ }^{1}$, M.N.A. Khan ${ }^{2}$ \\ Department of Computer Science \\ Shaheed Zulfikar Ali Bhutto Institute of Science and Technology \\ Islamabad, Pakistan
}

\begin{abstract}
This study analyses different techniques used for evaluation of various usability dimensions of software applications (apps) being used on the smartphones. The scope of this study is to evaluate various aspects of the usability techniques employed in the domain of smartphone apps. Usability assessment methodologies are evaluated for different types of applications running on different operating systems like Android, Blackberry and iOS, etc. Usability evaluation techniques and methodologies with respect to usability heuristics like field experiments, laboratory experiments models and usability standards are discussed in detail. The issues for evaluation of usability of smartphone apps are identified by considering limitations and areas of improvement outlined in the contemporary literature. A conceptual framework for usability evaluation of smartphone apps is also designed which would be validated through experimentation in the thesis work. This study is particularly useful to comprehend usability issues and their likely remedies to produce high quality smartphone apps.
\end{abstract}

Keywords-Usability; Jakob-Nielson usability heuristics; smartphone applications; ease of use; understandability; learning curve

\section{INTRODUCTION}

All Smartphones have become a daily use items and are popular among all the sections of society. Smartphones are now the most popular mobile technology. The statistical data show that one in each three citizens own a smartphones [1]. Smartphones are in fact modern mobile phones with an additional highly developed computing capability and connectivity. Their extensive modes for input are provided by a touch sensitive display. The most popular smartphones operating systems are Android, iOS, Windows phones and Blackberry. The quick and growing amount of smartphone apps on the Google play and Apple stores have facilitated and impelled the software experts to design applications of better quality in order to compete in the markets. There are numerous measureable aspects on the quality of software product and usability is one of the most significant aspects [1].

In the recent years, the introduction of numerous technologies has revolutionized our mode of communication, entertainment and completing daily routine tasks. Simultaneously, the method of digital convergence has resulted in the inventions of several devices like PDAs, smartphones, tablets etc. which are able to gather different forms of humancomputer interaction (HCI) in an integrated way. The HCI research community recommends considering different requirements when evaluating those applications, such as quantitative data (metrics), subjective evaluation (users' impressions) and context data (e.g. environment and devices' conditions.).

Usability is known as a significant quality dimension to evaluate the quality and usefulness of smartphone applications [2]. Usability is a quality attribute that assesses how easy and simple user interfaces are to use. According to Nielson, usability can be defined as a method for improving the design process. Usability is assessed on the basis of six dimensions which include learnability, memorability, efficiency, effectiveness, error rate and user satisfaction. Similarly, IEEE Standard.610.12-1990 defines usability as "The ease with which a user can learn to operate, prepares inputs for, and interprets outputs of a system or component." Usability dimensions affect four contextual factors which are users, technology, activity and environment. There are two classifications of usability each having different number of parameters. First classification includes effectiveness, efficiency and user satisfaction which are part of ISO 9241-11 standard. Second classification includes understandability, learnability, portability and attractiveness, and is known as ISO 9126-1 [3].

Table I illustrates six usability dimensions and their corresponding attributes. Brief description of these usability classification attributes are:

- Effectiveness: How accurately the user achieves the goals by using the app?

- Efficiency: How much resources are consumed to perform certain tasks?

- User Satisfaction: How do the users sense about their experience by using the applications?

- Learnability: Is the system easy to learn? Novice users should be able to complete basic tasks in a short period of time with minimum training.

- Memorability: The system is easy to remember/memorize. Users can return to it after a long period of time and complete tasks without retraining.

- Error Rate: If a user faces an errors while using the system, the system is capable to auto recovery. 
TABLE. I. USABILITY DIMENSIONS AND THEIR ATTRIBUTES

\begin{tabular}{|c|c|c|c|}
\hline \multicolumn{4}{|c|}{ Components of Nileson Usability Framework } \\
\hline $\begin{array}{l}\text { Usability } \\
\text { Dimensions }\end{array}$ & $\begin{array}{l}\text { Contextual } \\
\text { Factors }\end{array}$ & $\begin{array}{l}\text { Threshold values } \\
\text { for dimensions }\end{array}$ & $\begin{array}{l}\text { Classification } \\
\text { standards }\end{array}$ \\
\hline $\begin{array}{l}\text { Learnability } \\
\text { Memorability } \\
\text { Effectiveness } \\
\text { Efficiency } \\
\text { User } \\
\text { Satisfaction } \\
\text { Error Rate }\end{array}$ & $\begin{array}{l}\text { Users } \\
\text { Technology } \\
\text { Activity } \\
\text { Environment }\end{array}$ & $\begin{array}{l}\text { Time } \\
\text { Error rate } \\
\text { Number of apps } \\
\text { Number of users }\end{array}$ & $\begin{array}{l}\text { 1. ISO 9241-11 } \\
\text { Effectiveness } \\
\text { Efficiency } \\
\text { User Satisfaction } \\
\text { 2. ISO 9126-1 } \\
\text { Understandability } \\
\text { Learnability } \\
\text { Memorability }\end{array}$ \\
\hline
\end{tabular}

There are numerous ways to access the usability of smartphone apps. The prominent methods include expert reviews, user testing, field experiment, laboratory experiments, system usability scale measurement and user surveys. The questionnaire consisted of four parts, i.e., demographic (This part of the questionnaire gathered the demographic facts of participants, which includes age, gender, schooling, and earnings, and requested contributors to document their level of enjoy with smartphones and methods to connect to the mobile Internet. Participants' revel in with smartphones changed into measured with the aid of utilization hours in step with day and the total utilization duration) statistics, customers' preferences for the layout capabilities of smartphones, customers' reputation of smartphones, and customers' utilization behavior of smartphones [4].

Applying a heuristic evaluation approach using SMASH turned into shown to be effective in figuring out a huge percentage of the usability issues the aged customers confronted at the same time as interacting with a smartphone. The usability troubles had been not most effective because of UI design, a number of the problems had been due to problems of the elderly in performing the gestures which carried out to the corresponding undertaking; particularly, the "drag and drop", and "faucet and keep" gestures. Therefore, it is recommended that the usage of those gestures be removed or at least reduced.

Usability and user experience is a vital quality attribute for apps. The hedonic aspects such as fun, emotions and enjoyment are focused by user experience. Hedonic aspects meet up the universal needs, but they do not essentially have a utilization worth. User-ability is another aspect which is integrated with the user experience to determine whether the user felt pleasant or otherwise with the system during the inspection. An approach is used by the users in which they draw and write their hedonic aspects about the application [5]. This will leads to map the usability attributes on the usability heuristics as follows:

1) Visibility of the system (system should keep the user informed about all processes and state changes through feedback within a reasonable amount of time).

2) Match between system and real world (should speak users language instead of system oriented language).

3) User control and freedom (Allow users to undo and redo the previous tasks).

4) Consistency and standards (the user should be able to do things in a familiar standard and consistent way).
5) Error prevention (display appropriate mock up message).

6) Minimize the users memory load (interface should show the visible objects, actions and options).

7) Efficiency of use and performance (animated icons and transmissions should be displayed efficiently).

8) Aesthetic and minimalist design (avoid unwanted information, particularly the one that is out of context).

9) Help and documentation (easy to find different content and documentation help is available).

10) Help users identify, analyze and improve from errors (error should be displayed in user familiar language instead of system language). Customization and shortcuts (shortcuts are available for frequently used actions).

Physical interaction and ergonomics (should provide physical sense like buttons, position, etc.) [6].

If usability heuristics evaluations are conducted on a functioning/working product, the experts need to have some specific user tasks in mind to focus the inspection in the right direction [7].

Usability is an important factor as if an app is difficult to use then users would quit using that specific app. Usability testing in a right manner, at the right time and with the right observation would reduce the software risks of building the wrong product. For evaluation of smartphone applications, usability assessment is crucial so that developers can learn how to adopt them and consider the dynamicity of mobile scenarios.

Usability evaluation of smartphone applications is a potential research context that faces a number of challenges. These challenges emerge due to the unique restricted features of mobile phone such as limited bandwidth, varying environmental factors and unreliable network [4]. Additional challenges includes lack of usage-based testing and response, limited focus on interface architecture, navigation ignorance and connectivity, restricted resources and web connectivity issues. The technical capabilities of mobile apps and achieving high level user satisfaction are crucial for the success of mobile apps. Hence, usability testing of mobile apps is mandatory process to ensure that mobile apps are practical, effective and easy to use [8].

Because of the significance, a large number of usability guiding principles have been modeled to support the structure of usable applications. These guiding principles are specially proposed for web-based and desktop applications. Due to the mobility nature of smartphones devices, the smartphone apps are different in many ways from the conventional applications [9]. To date, the guiding principles for usable smartphone applications are isolated and limited. This adds difficulty and complexity to evaluate the usability of smartphone apps [10].

This aim of this study is to explore the various usability dimensions and the corresponding issues that need to be considered while designing and evaluating mobile apps. In this study, we evaluate different usability dimensions along with their testing parameters that are necessary to be adhered to ensure better quality of the mobile apps and their userfriendliness. 


\section{LITERATURE REVIEW}

Moumane et al. [3] present empirical evaluation for the use of software quality standards ISO-9126 in mobile environments and highlight issues related to the software such as user guides, use of simple data entry procedure and existence of online help. The study performs hardware based evaluation such as display resolution, memory size and screen size by using ISO-9241 and ISO-5062 standards benchmark. The authors analyze two usability evaluation standards for mobile apps including ISO-9241 and ISO-25062.ISO standard 9241 is a base quality model and includes efficiency and satisfaction as the usability dimensions. The level of user satisfaction against three usability factors were evaluated as $62 \%, 33 \%$ and $20 \%$ respectively. ISO standard 25062 includes two usability factors reliability and portability. The proposed framework was compared with ISO standard 9126 on the basis of three usability measures efficiency, effectiveness and satisfaction by conducting a survey.

Sorber and Kortum [11] addressed the subjective usability of a large number of mobile apps for both tablets and phones across Android and Mac operating systems and target the consistency measure in this context. The objective of the study is to propose a baseline for usability measures for mobile apps. The author describe the usability on a large number of mobile apps for both tablets and phones across Android and Mac operating systems; characterize these results on system usability scale and describe the usability measures for consistency. The proposed solution is used only on small scale. Future work could be examining the usability of mobile apps in a more formal laboratory based environment.

$\mathrm{Lu}$ and Wei [12] analyze the level of enjoyment, mobility use towards the persistence use of smartphone apps. The goal of study is to revise the IS (Information System) continuance model to highlight the role of enjoyment and mobility of user perception towards the continuance of user intention to use the smartphone application. The IS continuance model is based on the ECT(Expectation Theory of Continuance).ECT is mostly used in the literature for consumer behavior to analyze the consumer satisfaction, service marketing in general and postpurchase behavior(e.g., repurchase, complaining), (Anderson and Sullivan1993; The usability measure include satisfaction, performance expectancy, continuance intention, post usage attitude and effort expectancy. The study defines the context of smartphone apps broadly and data were analyzed on a smaller scale. Future work could be to validate the proposed model on large scale by using some other design methodology.

Baek and Yoo [14] evaluate the usability attributes for "branded mobile applications" to measure and conceptualize the underlying scope of usability. The research objective is to propose and examine the measurement tool to explore the perceived level of usability application. Study proposed the usability factors as user friendliness, omnipresence speed, fun and personalization, for app and proposed a valid and reliable exploration of the usability application that included user perceptions. The study develops the usability scale development model. Variable of interests in data gathering were explored and measured using self-report survey. Future study could be conducted to validate the usability scale for usability using a randomly selected model from other mobile user population based on different types of branded apps like native, hybrid and desktop apps.

Lorusso et al. [15] address the usability and learnability of NFC based application. This research aims to explore the user satisfaction, usability, learnability and quality of the interaction between the children who have language disorder and the system application. Autonomy level, feedback, satisfaction and learnability are usability variables. Limited numbers of activities are offered by the selected prototype/hypothesis. As a future dimension, a systematic research should be conducted in educational environment.

Hussain et al. [16] explore the usability on kindle app for smartphone platform. For this purpose usability attributes as visibility, efficiency, satisfaction and enjoyability. Study proposed a descriptive based statistical methodology to evaluate the usability attributes. In laboratory based experiment 15 participants were chosen randomly with different age groups ranges as 18-29, 30-39 and 40-49. Five tasks were performed by the participants. One minute is set for execution of task. The test session is recorded by video camera. The front screen recorded the emotions, time, error and navigation from one page to another page of the users. A post-test questioner is given to the users. There were three measurements such as time, error, and frequency. Quantitative data is gathered by the test results and analyzed with the SPSS. Descriptive statistics include Min, Max and Standard deviation were used to analyze and present the resulted data, these resulted values are mapped on Likert scale which has values as strongly agree, Disagree, Natural, Agree and Strongly disagree. Future studies could be conducted on large no of participants when the sample is projected to the large population.

Nascimento et al. [5] explore the usability by addressed the relationship between user experience and usability. Study proposed a technique "userability" to evaluate the usability for mobile applications. The userability is the integration of user experiences and usability which helps to the developer designer as well as non specialist in the domain of human computer interaction. Proposed methodology consists of two further steps as heuristic evaluation for usability and $3 \mathrm{E}$ method. The 3E method stands for Expressing Emotions and Experiences. Study define the ten aspects from heuristic evaluation and from $3 \mathrm{E}$ method the two questions are finalize for evaluation as "what users feel regarding ten heuristics aspects and "what are the improvements did users feel for this aspects. Satisfaction is the main attributes in $3 \mathrm{E}$ and ten heuristic aspects for this evaluation emojis of face expression is used. The satisfaction attribute is scaled on questioner as unsatisfied, moderately satisfied, little satisfied very satisfied and very satisfied. The proposed methodology phases include the steps as Training, Application scope, scope of the activities, qualitative analysis of inspection questioner Detection issues and Data analysis. Five applications are used measurement are time, no of errors, no of duplicated issues, suggestions and duplicated suggestions. Grounded Theory method is used for validation to perform data coding.

Salman et al. [6] evaluate the usability of user interface for smartphone applications used by elderly. Heuristic evaluation 
methodology is used for this purpose. Issues are highlighted during the expert testing sessions. Identified problems are classified into different categories as appearances, language, dialogue and information. These classifications are further divided into sub categories and proposed solution for user interfaces. From heuristics evaluation two heuristics are frequently violated as minimize the user memory load and match between system and real world. The test session is performed by the 5 experts who have different age groups and have different qualification in human interaction domain on smartphone model J7. By the end of the results, 27 problems are highlighted extracted from a checklist. In future studies, a think aloud session would be conducted with the elderly participants at the time of development and design a prototype after getting their relevance feedback.

Lee and Lee [13] evaluate the usability attributes for augmented reality mobile application. This research aims to develop a tool for creating user-based design interfaces in mobile augmented reality (MAR) education and check list for usability with factor analysis and reliability. Examine the usability attributes of multimedia AR and to develop a usability evaluation tool through concretization. This study examined the usability attributes like learnability, ease of memory, usage convenience and satisfaction. The evaluation items collected from existing research were used as basic data for developing the usability evaluation checklist survey was conducted with 122 experts, and after factor analysis and reliability analysis, the final checklist for each usability evaluation item was prepared. Affordance and presence are main measures of reliability with cognitive affordance, sensible affordance, and physical affordance .proposed the usability evolution tool with focus group interview, factor analysis and reliability on evolution question. Proposed evolution steps do not validate with the existing model. Future work could be to validate the proposed usability steps on large no of applications in formal laboratory experiment.

Liu et al. [17] address the usability aspects under acceptance and usage behavior of smartphone applications. Factors are analyzed regarding acceptance and behavior. Questioner was developed which is filled by 842 participants. Acceptance is measured as usefulness, ease of use, and intention of use. Nine factors are explored for acceptance as element for design interface, physical smartphone characteristics, feedback for touch screen, display screen, connectivity and application. A questioner is constructed comprising four parts demographic information (gender, age, education and income etc.), user preferences, features of smartphone (icon size, icon color, shape, font size, character spacing, etc.), users acceptance and their usage behavior (task based like shopping Skype chatting, etc.). Data were collected an online survey of smartphone users from a Chinese website. For data analysis EFA (exploratory factor analysis) is used to detect the critical design features factor and CFA (confirmatory factor analysis was) is used to check reliability and validity for measurement constructs.842 participants including 378 male and464 are female having at least 4 years' experience for using smartphone, age groups ranges as 20 to 51 years. Age attribute is divided into categories then find frequency of each age groups among male and female and result the mean and standard deviation. These studies result is generating by considering the android operating system on apple or any other operating system results may differ or better just because of hardware limitation as display resolution speed, etc.

\section{CRITICAL ANALYSIS}

In this study after reviewing the reviewing the literature the usability assessment methods for testing are briefly described and critically evaluate the literature to explore various usability dimensions as well as corresponding challenges that need to be considered while designing and evaluating the mobile applications. By considering the various usability dimensions, attributes, performance measures, contextual factors, testing parameters, proposed model and validation model as well.

The attribute of the critical table are Problem addressed, Usability dimension, Implementation method, platform used for application, standard model for comparison or validation purpose, mapped with Nielsen findings for user interface named as SMASH (smartphone usability heuristics) and limitation or future findings.

\section{Key Challenges}

Since mobile technologies are used in every field, smartphone apps play a vital role for their success. So usability is a crucial factor to achieve the quality goal but usability testing of smartphone apps faces number of challenges like:

Connectivity [3,8]: The slow, unchangeable network association with small size bandwidth is an ordinary difficulty for smooth execution of smartphone apps. The difficulty mainly affects loading time and worth of stream media.

Small screen size [3,8]: The diversity of element ratio and pixel solidity can be massive.

Different display resolutions [4]: As different display resolution may produce different usability evaluation results, short resolution can disgrace the quality of information display on mobile devices.

Context of mobile [14]: It may illustrate as any statistics that differentiates a situation linked to conversation between user, apps and the encompassing historical past. It naturally consists of location identities of close by humans, gadgets and environmental basics that could divert person awareness.

Capability and Limited processing power [15]: Some smartphone apps want large quantity of remembrance to GUI assist which include three dimensional apps.

Lesser focus on navigation [14]: Interface structure play a critical position to get the consumer pride stage. On interface structure there's a number of unnecessarily links or buttons which burdened the consumer and frustrated pop-up messages on each second.

Lack of use testing and response $[8,15]$ : The most important challenges in usability testing is the lack of user testing and their feedback during the design evaluation process. There is a need to get the acknowledgement from user to determine their needs, intentions, usability obstacles through a descriptive or statistical measure. 
Not strengthening the engagement loop [3,15]: Designer should carefully design the app by getting the user experiences, their preferences for the application by performing the tasks and taking the feedback.

\section{CONCEPTUAL FRAMEWORK}

Conceptual framework for usability evaluation of smartphone is described in Fig. 1.

Conceptual framework is the approach to represent a general concept, that guide people appreciate or reproduce the domain of that model. Conceptual models illustrate the relations between factors and the stream of data or processes. A conceptual model comprises the four fundamental characteristics as the potential reward to implement a theoretical model are numerous, but mostly depend on the own capability to invent a well-built model in the primary place. The key rewards of theoretical model include.

Description: The above conceptual model is designed for usability testing of Smartphone applications. The conceptual model comprises of six steps as selection of usability attribute, usability evaluation based criteria, fetch threshold values for usability attributes, select the application for usability testing, design test case generation on the basis of usability criteria, Test execution and check the criteria meet for validation. Three mini table which support to select the attributes as (learnability, efficiency, memorability, error, satisfaction and effectiveness) standards and models to follow for the basic initiation process as (ISO 9241-11 and ISO 9126-1) and measuring parameters as (time, error rate, number of users and number of applications laboratory or field experiment, and contextual factor as well) for usability testing as stored in a tabular form. All these steps should be followed before designing the smartphone application. Arrows show the flow of data, boxes indicate the main steps, diamond symbol show the decision in form of "Yes" or "No".

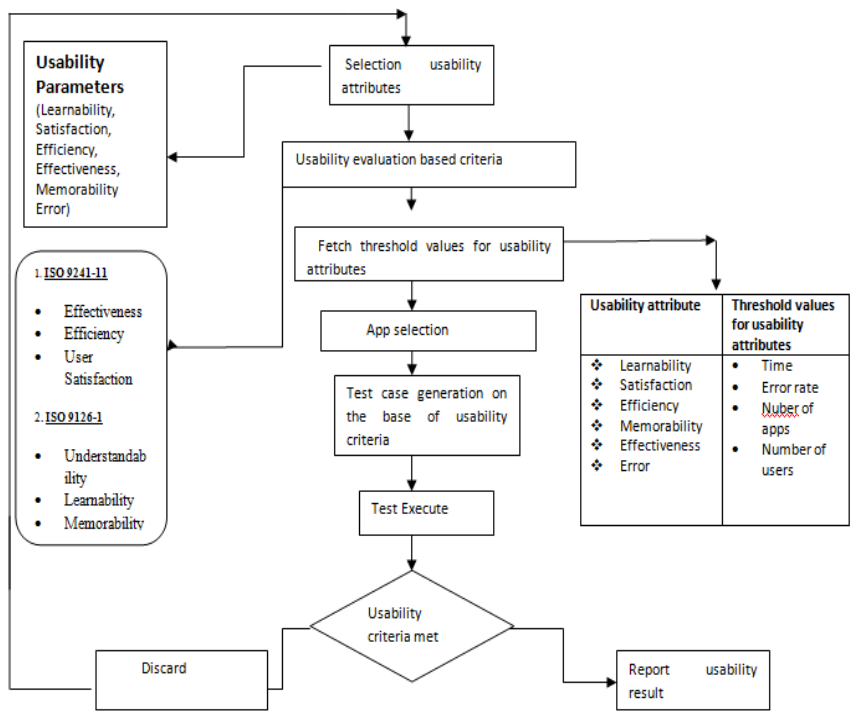

Fig. 1. Conceptual Framework for usability Evaluation.
Selection usability attributes: In the first step usability selection is the initiation process to usability of smartphone app. The user selects the usability dimensions as per requirement or his scope of the work. The usability attribute are stored as a list in the tabular form as learnability efficiency memorability, error, satisfaction and effectiveness, effectiveness, efficiency and user satisfaction are mostly and commonly used as per literature review. so selection the usability is a vital for further processing.

Usability evaluation based criteria: In this phase the usability evaluation based criteria is chosen to access which standards or models are the based for further processing. The criteria list for usability testing as standards and model like (ISO 9241-11 and ISO 9126-1) are stored in the tabular form with their different usability parameters.

Fetch threshold values for usability attributes: In the third step after selection of usability dimension and criteria the threshold values are selected. The threshold values must be defined to meet or to compare the mature results like volume of expected traffic, error rate, time etc. The threshold values may be quantitative or statistical measure like time, frequency, min, max, error rate, number of users and number of apps, etc. The threshold measurement values are fetched from a table in which these values are listed.

App selection: After set the usability dimension their testing parameters there is need to implement on an application. The application is selected on bases of the nature of the testing. The contextual factors as technology, type of users, activity and environment etc. play a vital role for selecting the application. Which platform, operating system is used for testing this will be set in this phase.

Test case generation on the base of usability criteria: In the 4th step when the application, platform operating system is selected then there's a need to design a prototype for testing. Prototype is usually used to estimate a brand new design to growth accuracy with the aid of device examination and consumer. A prototype is an premature pattern, reproduction or freed from a product built to check a belief or procedure or to carry out as a thing to be simulated or found out from. This can be questioner, laboratory, field experiment or in a controlled environment.

Test Execute: After selecting the platform, the operating system and the application the designed prototype is being executed by the users. The method of execution could be a pre or post questioner, advance techniques like eye tracing and facial recognition is used to get user experiences, and emotions " 3 E method" is used which explore the expressing emotions and experiences.

Usability criteria met: In the last step execution of the designed prototype and calculate the result after the processing. These results are validated with an existing model or prototype result. Results are noted and validate with the models that is chosen on the second step as criteria for usability evaluation. If the criteria meet the with the model or show better 
performance and then results are documented in the form of report But if the criteria is not meet then move back to the step one and repeat the process by changing the attributes or performance parameters for good results. evaluation results are met with the defined criteria or enhancement is occur while comparing then report is generated, if the evaluation results are not met with the pre-defined criteria.

\section{CONCLUSION AND FUTURE WORK}

Usability is recognized as a significant quality dimension to determine the success of mobile applications. This study highlights the techniques which are being applied to evaluate the usability of smart phone applications. Usability assessment methodologies are evaluated for different types of applications running on different operating systems like Android, Blackberry and iOS etc. Assessment methods of usability testing are discussed in a great detail to explore various usability dimensions as well as corresponding challenges that need to be considered while designing and evaluating the mobile applications. Specifically, the study conducted a critical review of various usability dimensions, attributes, performance measures, contextual factors, testing parameters, proposed model and validation model as well. The prominent challenges identified in this study include: connectivity, small screen size, different display resolution, information input method, context of mobile, capability and limited processing power, navigation ignorance, no focus on interface architecture etc. A conceptual framework for usability evaluation of smartphone apps is also designed which would be validated through experimentation in the thesis work. This study is particularly useful to comprehend usability issues and their likely remedies to produce high quality smartphone apps. The study provides a conceptual framework for usability testing of smartphone applications. Future work could be conducted to validate this model in a formal manner.

\section{REFERENCES}

[1] B.C. Zapata, J.L. Fernández-Alemán, A. Idri and A. Toval, "Empirical studies on usability of mhealth apps: a systematic literature review," Journal of medical systems, 39(2), 2015.

[2] R. Inostroza, C. Rusu, S. Roncagliolo, V. Rusu and C. Collazos, "Developing SMASH: a set of smartphone'susability heuristics," Computer Standards \& Interfaces, 43 pp.40-52, 2016.
[3] K. Moumane, A. Idri and A. Abran, "Usability evaluation of mobile applications using ISO 9241 and ISO 25062," Springerplus, 5(1), 2016.

[4] H. Rahmat, H. Zulzalil and A. Abdulghani, "An approach towards development of evaluation framework for usability of smartphone applications," Malaysian Software Engineering Conference (MySEC), pp. 178-182, 2017.

[5] I. Nascimento,W.Silva, B. Gadelha and T.Conte, "Userbility: a technique for the evaluation of user experience and usability on mobile applications," Human-Computer Interaction, 37:3, 372-383,19 June 2016.

[6] H.M. Salman, W.F. Wan and S. Sulaiman, "Usability evaluation of the smartphone user interface in supporting elderly users from experts' perspective," IEEE Access, 6, pp. 22578-22591, 2018.

[7] D. Quiñones and C. Rusu, "How to develop usability heuristics: A systematic literature review," Computer Standards \& Interfaces, 53(2017): 89-122, 2017.

[8] B.A. Kumar and P. Mohite, "Usability of mobile learning applications: a systematic literature review," Journal of Computers in Education, 5(1), pp. 11-17, 2018.

[9] R.Alturki, V.Gay and R. Alturki, "Usability attributes for mobile application: a systematic review," 7th International Conference on Computer Science, Engineering \& Applications, 2017.

[10] D. Zhang and B. Adipat "Challenges, methodologies, and issues in the usability testing of mobile applications," International Journal of Human-Computer Interaction, 18(3), pp. 293-308, 2005.

[11] P. Kortum and M. Sorber, "Measuring the usability of mobile applications for phones and tablets," International Journal of HumanComputer Interaction 31(8), 518-529, 2015.

[12] J. Lu, C. Liu and J. Wei, "How important are enjoyment and mobility for mobile applications?," Journal of Computer Information Systems, 57 (1), pp. 1-12, 2017.

[13] W-H Lee and H-K Lee, "The usability attributes and evaluation measurements of mobile media AR (augmented reality)," Cogent Arts \& Humanities, 3(1), 2016.

[14] T.H. Baek and C.Y. Yoo, "Branded app usability: conceptualization, measurement, and prediction of consumer loyalty," Journal of Advertising, 47(1), pp. 70-82, 2018.

[15] M.L. Lorusso, E. Biffi, M. Molteni and G. Reni, "Exploring the learnability and usability of a near field communication-based application for semantic enrichment in children with language disorders," Assistive Technology, 30(1), pp. 39-50, 2018.

[16] A. Hussain, E.O. Mkpojiogu, J.A. Musa and S. Mortada, "A user experience evaluation of Amazon kindle mobile application," In AIP Conference Proceedings (Vol. 1891, No. 1, p. 020060), AIP Publishing, 2017.

[17] N. Liu and R. Yu, "Identifying design feature factors critical to acceptance and usage behaviour of smartphones," Computers in Human Behaviour, 70(2017), pp.131-142, 2017. 